\title{
Counselor and Drug Detox Inpatient Verbal Behaviors in a Single Session of Motivational Interviewing and Subsequent Substance Use-Related Patient Outcomes
}

\author{
Anne H. Berman ${ }^{1,2}$ - Helena Lindqvist ${ }^{1,3,4}$. \\ Håkan Källmén ${ }^{1}$ • Natalie Durbeej ${ }^{1}$. \\ Ulric Hermansson $^{1,2} \cdot$ Lars Forsberg $^{5}$
}

C The Author(s) 2018. This article is an open access publication

\begin{abstract}
Effects of counselor and client verbal behaviors, in a single motivational interviewing (MI) session during inpatient detoxification, were evaluated in relation to 17 study participants' self-reported drug use 3 months later. Also, counselor-to-client transitions in the single MI session were explored for 24 participants, using the Motivational Interviewing Sequential Code for Observing Process Exchanges (MI-SCOPE) coding instrument. Surprisingly, counselor neutral/double-sided reflections predicted lower levels of drug use $(r=-$ $0.42)$, higher treatment interest $(r=0.40)$, and self-efficacy for abstaining from drug use when experiencing positive feelings $(r=0.48)$ and negative feelings or craving $(r=0.40)$. Client change talk predicted motivation to change $(r=0.42)$ and perceptions of negative aspects of drug use $(r=0.44)$, while higher levels of client sustain talk predicted lower levels of drug use $(r=-0.45)$ and lower interest in treatment $(r=-0.68)$. The counselor-client transition analysis showed an odds ratio (OR) of 13.84 (95\% CI 9.75; 19.66) for client change talk in response to counselor reflections on change talk (RCT), and an OR of 55.98 (95\% CI 26.53;
\end{abstract}

Anne H. Berman

anne.h.berman@ki.se

1 Centre for Psychiatry Research, Department of Clinical Neuroscience, Karolinska Institutet, Norra Stationsgatan 69, 7th floor, Norra Stationsgatan 69, SE-11364 Stockholm, Sweden

2 Stockholm Center for Dependency Disorders, Stockholm Health Care Services, Stockholm County Council, Stockholm, Sweden

3 MIQA-group, Centre for Psychotherapy Education \& Research, Stockholm Health Care Services, Stockholm County Council, Stockholm, Sweden

4 Stockholm Health Care Services, Stockholm County Council, Stockholm, Sweden

5 Department of Psychology, Uppsala University, Uppsala, Sweden 
118.12) for client sustain talk in response to counselor reflections on sustain talk (RST). Future research should increase sample size and extend follow-up.

Keywords Detoxification · Hospital $\cdot$ Motivational interviewing $\cdot$ Drug use $\cdot$ Counselor $\cdot$ Client

A significant challenge facing treatment providers for problematic substance users is to mobilize the client's motivation and stimulate behavioral change within a short period of time. This challenge is particularly relevant within inpatient detoxification care, when clients are in crisis and in treatment for several days. Research on psychosocial interventions in the detoxification setting is sparse, but the evidence available suggests that receiving three motivational interviewing (MI) sessions increases the proportion of alcohol detoxification patients who participate in 12-step groups 2 months later (Schilling et al. 2002) and that receiving a single MI session positively affects drug detoxification patients 3 months later, in terms of increased awareness of the positive effects of drugs as well as increased self-efficacy regarding the capacity to abstain from drugs in connection with positive feelings (Berman et al. 2010). Brief MI interventions thus may have potential for eliciting small but significant effects in the detoxification setting.

The positive findings regarding the effects of such minimal interventions raise the question of exactly what aspects of MI might be key to effecting healthy behavioral change in clients. A causal chain of behavioral links has been proposed and analyzed, suggesting that effective MI treatment is, to a certain extent, mediated by therapist behavior on the one hand, and client behavior on the other, where the association with substance use outcomes is $r=0.13$ for therapist behavior and $r=0.23$ for client behavior. The link between therapist and client insession behavior is stronger, $r=.46$ (Apodaca and Longabaugh 2009, p. 711).

Counselor characteristics and in-session behavior can be conceptualized as relational or technical, where the former concerns interpersonal aspects of the counselor-client interaction, and the latter concerns the interrelationship between counselor verbal behavior and client responses (Miller and Rose 2009). Both relational and technical aspects of therapist behaviors have been termed "common" or "non-specific" factors, difficult to measure and confounding methodological or procedural content of treatment interventions. Miller and Moyers (2015) have argued for the importance of specifying and researching such factors, an endeavor made possible by the series of counselor and coding systems developed for evaluation of MI sessions, where the Motivational Interviewing Sequential Code for Observing Process Exchanges (MI-SCOPE) (Glynn et al. 2012; Moyers et al. 2009) has been recommended for MI process research (Dobber et al. 2015).

Prior research has investigated links between client in-session verbal behavior and outcome, counselor in-session behavior and client substance use outcome, and the interrelation between counselor and client verbal behaviors, among college students with heavy episodic drinking (Vader et al. 2010), adolescents with problematic substance use (Baer et al. 2008), and nontreatment-seeking men with overconsumption of alcohol (Gaume et al. 2008a). Findings suggested that MI-consistent counselor behavior was more likely to be followed by client change talk, where client verbal exploration of change also generated more MI-consistent counselor behavior (Gaume et al. 2008b); high frequency of clients' in-session expressed reasons in favor of change predicted greater abstinence from alcohol or illicit substances (Baer et al. 2008); and the quality of MI counseling affected change talk levels among heavy college student drinkers, and their related drinking outcomes (Vader et al. 2010). To our knowledge, no 
studies focusing on counselor and client verbal behaviors with inpatients in hospital detoxification units have been published. This article explores the relationship between MI counselor behavior, clients' in-session verbal behavior, and client behavioral outcomes, by investigating drug detoxification counselors' and inpatients' in-session MI-related verbal behavior, and associations with patient self-efficacy, motivational levels, and self-reported drug use. The data were originally collected in a randomized study of the effects of in-hospital single-session MI on drug detox inpatients' self-efficacy, motivational levels, and self-reported drug use 3 months after inpatient treatment (Berman et al. 2010). However, the state of the art in the area of valid analysis of sequential counselor-client utterances has only recently advanced to a point where it appeared worthwhile to analyze utterance transitions in the small sample of inpatients who participated in our original study. As data accumulate on the mechanisms and processes of MI conversations, the complexity of interacting factors is the focus of an ongoing discourse (Pace et al. 2017), to which this study is an additional contribution from a care context where knowledge about MI mechanisms has not yet been charted.

\section{Specific Aims and Research Questions}

The relationship between MI counselor language and client language was explored during a single MI session delivered at a drug detoxification hospital unit. The predictive value of such counselor- and patient language for client substance use outcomes after 3 months was also investigated. More specifically, we sought to answer the following research questions:

1. Does counselor in-session language predict client substance use outcomes 3 months following the MI single-session intervention?

2. Does client in-session language predict client substance use outcomes 3 months following the MI intervention?

3. Is counselor in-session language associated with client in-session language?

Substance use outcomes, all self-reported, were evaluated in terms of self-efficacy for abstaining from alcohol or drug use, motivation to change based on positive and negative aspects of drug use and treatment readiness, and drugs used. Our specific hypotheses were that (a) MI counselor focus on client change talk would predict client self-efficacy for abstaining from alcohol and drugs; (b) client change talk would predict client motivation to change as well as, possibly, substance use outcomes; (c) counselor behaviors focusing on client change talk would precede client change talk more frequently than counselor behaviors focusing on client sustain talk.

\section{Method}

\section{Procedure and Participants}

Recruitment of study participants is described in detail elsewhere (Berman et al. 2010). To summarize briefly, 213 participants were recruited among inpatients at a Stockholm hospital drug user detoxification unit between 2006 and 2008. Patients who agreed to participate filled in questionnaires on drug use, self-efficacy for abstaining from alcohol or drug use, motivation 
to change, type of drugs used, positive and negative aspects of drug use, and treatment readiness for drug use (see measures below). Following completion of questionnaires, participants were randomly allocated to one MI session or to treatment-as-usual (TAU) except when only one or two patients accepted participation, in which case they were automatically allocated to MI sessions. The MI sessions were held by MI-trained unit staff on the evening or night following recruitment and lasted 45-120 min. The target behavior for each MI session was left open to definition by the client, but it generally concerned reduction of problematic drug use. Participants in the TAU condition received standard inpatient detoxification care, consisting of medication as needed to control symptoms of abstinence, regular meals, a hospital bed, and access to leisure activities, as well as ad hoc conversations with unit staff. The unit did not provide systematic counseling, but offered referral to social or psychiatric services when needed. Three months after recruitment, participants were contacted and asked to fill in follow-up questionnaires. The final analyzable sample consisted of 87 participants, 66 men and 21 women, with an average age of 37.2 (SD 10.4), and 77\% reporting both alcohol and drug use, and $23 \%$ reporting only drug use. Each participant received two cinema gift vouchers following delivery of completed follow-up questionnaires. The Stockholm Regional Ethical Vetting Board approved the study (ref. no. 2005/429-31/3).

Of the 47 patients who received MI, 12 were lost to follow-up. Of the 35 remaining patients analyzed in our original study (Berman et al. 2010), 11 were excluded from this study due to technical difficulties, leaving 24 participants (69\%) for whom client commitment language as well as counselor MI-adherence were coded. Complete outcome data were available for 17 of these participants. Analyses thus used 24 sessions to analyze transitions between counselor and client language, and $17 \mathrm{MI}$-sessions to analyze associations with 3-month client substance-userelated outcomes.

\section{Measures}

Three classes of measures were used: one to assess the quality of therapists' adherence to MI (1), the second to evaluate client outcomes over time (2), and the third to evaluate the relationship between counselor and client utterances. The first two classes of measures are described fully in our previous article (Berman et al. 2010) and are summarized briefly below. The third class of measures is then described in detail.

1. To assess the quality of MI-adherence (e.g., reflective listening, MI-adherent utterances), we coded the sessions according to the Treatment Integrity Scale, version 3.0 (MITI 3.0; Moyers et al. 2007b). Although the MI sessions lasted between 45 and 120 min, according to standard practice, only the first 20-min segments of each session were MITI-coded. Three senior coders at the Motivational Interviewing Quality Assurance (MIQA) laboratory at Karolinska Institutet performed the coding. The coders' inter-rater reliability, calculated as intra-class correlations, was in the good to excellent range (Cicchetti 1994). The MITI coding indicated satisfactory MI-adherence (Berman et al. 2010).

2. Client outcome was measured in terms of self-reported drug use (a), in-depth aspects of drug use (b), and self-efficacy for abstaining from alcohol and drug use (c). The measures used were as follows.

(a) Drug use was measured with the Drug Use Disorders Identification Test (DUDIT; Berman et al. 2005), which comprises 11 items, 4 on illicit drug use and 7 on various 
drug-related problems. A review of 16 studies in a variety of samples showed internal consistency reliability expressed in terms of Cronbach's $\alpha$ between 0.74 and 0.97 , where most studies reported an $\alpha$ of over 0.90 (Hildebrand 2015). In the current sample $(n=$ 24), $\alpha$ was 0.86 .

(b) In-depth aspects of drug use were measured using the Drug Use Disorders Identification Test-Extended E (DUDIT-E; Berman et al. 2007), which includes 54 items divided into four subscales: current drug use (DUDIT-Ed; D), positive aspects of drug use (DUDITEp; P), negative aspects of drug use (DUDIT-En; N), and treatment readiness (DUDITEt; T). Based on the P, N, and T scales, a Motivational Index (MotInd) can be calculated to measure level of motivation to change by dividing the $\mathrm{N}$ score by the $\mathrm{P}$ score and then multiplying by the T score. Internal consistency reliability in terms of Cronbach's $\alpha$ can be calculated for the N, P, and T scores, and has been reported as ranging from 0.88 to 0.95 for the $\mathrm{P}$ score, 0.88 to 0.93 for the $\mathrm{N}$ score, and 0.72 to 0.81 for the $\mathrm{T}$ score (Berman et al. 2007). In this sample, Cronbach's $\alpha$ was 0.83 for $\mathrm{P}$ scale, 0.76 for $\mathrm{N}$ scale and 0.65 for the $\mathrm{T}$ scale.

(c) Self-efficacy regarding abstention from alcohol and illicit drugs was measured using 12item versions of the Alcohol Abstinence Self-Efficacy Scale (AASE; DiClemente et al. 1994; McKiernan et al. 2011) and the Drug Abstinence Self-Efficacy Scale (DASE; see Berman et al. 2010). Items from both the AASE and the DASE can be divided into subscales measuring self-efficacy regarding abstinence from alcohol and illicit drugs in connection with positive feelings, negative feelings, physical discomfort, and craving (AASEpos, AASEneg, AASEphy, AASEcrav and DASEpos, DASEneg, DASEphy and DASEcrav, respectively). Internal consistency reliability for the 12-item AASE has been reported as ranging from Cronbach's $\alpha$ values of 0.88 to 0.92 in a sample of 126 inpatients and outpatients receiving addiction treatment (McKiernan et al. 2011), but we are unaware of any prior published reports of Cronbach's $\alpha$ for the 12-item DASE. In this sample, Cronbach's $\alpha$ values were 0.95 and 0.90 for the AASE and DASE, respectively.

3. Assessment of client and counselor sequential utterances was performed by coders at the MIQA laboratory at Karolinska Institutet, using the Swedish version of the Motivational Interviewing Sequential Code for Observing Process Exchanges (MI-SCOPE; Forsberg et al. 2014). The MI-SCOPE is derived from two other coding systems: the Motivational Interviewing Skill Code (MISC; Moyers et al. 2003), and the Commitment Language Coding System (Moyers and Martin 2006) and was developed to code both client and counselor language in transcripts of recorded MI sessions, with a particular focus on the sequential information between the two parts.

Two senior coders from the MIQA laboratory coded all sessions using the MI-SCOPE. One coder parsed all sessions (divided all client and counselor talk into separate utterances) and the other coder then assigned each counselor- and client-parsed utterance one of the 45 MI-SCOPE codes (16 client codes and 29 counselor codes) (Martin et al. 2005). For the purpose of the analysis conducted in the current study, all client variables were collapsed into three categories and all counselor variables were collapsed into seven categories (Forsberg et al. 2014; Martin et al. 2005). The three client categories concerned change talk, sustain talk, and neutral talk. Thus, all client utterances related to the target behavior were combined into a change talk category (CT; language that favors change) or a sustain talk category (ST; language that favors status quo). All 
remaining client utterances were collapsed into a follow/neutral and ask category (FN). The seven collapsed counselor categories were as follows: all questions evoking positive aspects of the target behavior, in this case drug use (QPOS); all questions evoking negative aspects of the target behavior (QNEG); all neutral questions (QNEUT); all reflections on change talk (RCT); all reflections on sustain talk (RST); all neutral or double-sided reflections (REF); and all other counselor variables (i.e., general information, structure) combined into one category (Other). MIconsistent counselor utterances (support, affirm, emphasize control, permission seeking), as well as MI-inconsistent utterances (advise, confront, direct, opinion, warn), have previously been analyzed as separate categories (e.g., Gaume et al. 2008a; Moyers et al. 2009). The presence of these variables in our data set was low, however. For this reason, these utterances were not analyzed separately but were instead included in the "Other" category. The MI-SCOPE has demonstrated moderate to good inter-rater reliability in previous research (Moyers and Martin 2006; Moyers et al. 2009).

\section{Statistics}

Pearson product-moment correlations were calculated to evaluate the associations between MIcounseling variables, client language, and self-report variables. The squared correlation coefficients of determination were considered as related when corresponding to $10 \%$ or more common variance, i.e., equivalent to a Pearson correlation $(r)$ of \pm 0.32 and higher. Cohen has suggested the following interpretation of effect sizes for coefficients of determination $\left(r^{2}\right): 0.01$ small effect; 0.06 medium effect and 0.14 large effect (Cohen 1988); we have thus opted to report effects at the medium level and above. All analyses were performed with SPSS version 22. When calculating sum scores of scales with several items, actual responses were summed; this means that missing data on single items (internal dropout) were considered as zero responses. This procedure gives a larger $n$ but generally weaker correlations.

We evaluated the relationship between counselor and client utterances by calculating transition probabilities (TPs) with GSEQ software, version 5.1 (Bakeman and Quera 2011, 2015, Bakeman et al. 2009, Quera et al. 2007), based on the number of observed and expected transitions. The minimum number of expected transitions should be at least 3 to be able to compute reliable transition probabilities (Martin et al. 2005). The TPs were examined at lag 1 (i.e., the probability that a specific client utterance occurs immediately after a specific counselor behavior) and all 24 sessions were pooled in the analysis. Odds ratios (ORs) with $95 \%$ confidence intervals $(\mathrm{CI})$ were calculated to assess the probability of a specific client utterance (e.g., change talk, CT) occurring directly after a specific counselor behavior (e.g., RCT), compared with the probability of this specific client utterance occurring after any other counselor behavior. An OR greater than 1 indicates that the specific client utterance is more likely to occur in the presence of the specific counselor behavior than in its absence, and an OR lower than 1 indicates that the client utterance is less likely to occur in the presence of the counselor behavior than in its absence (Bakeman and Quera 2011).

\section{Results}

The results are presented in sequential order, following the research questions specified above. All correlations reported are between counselor and client verbal behaviors during the MIsession, and specific dimensions of client self-report questionnaires measured 3 months after the MI-session. 


\section{Counselor In-session Language and Client Substance Use-Related Outcomes}

We found a positive correlation between QPOS and DUDIT-En $(r=.41)$ suggesting that questions evoking positive aspects of drug use were associated with experiencing negative aspects of drug use at follow-up. Furthermore, QNEG was negatively related to DUDIT-Et $(r=-0.47)$ and DASEneg $(r=-0.33)$ indicating that questions evoking negative aspects of drug use were associated with low treatment interest and low self-efficacy in connection with negative feelings. Interestingly, RST was negatively related to DUDIT-Et $(r=-0.43)$ and positively associated with the motivational index $(r=0.42)$, demonstrating that reflections on sustain talk were associated with low treatment interest but moderate motivation to change. Moreover, there was a negative correlation between REF and DUDIT-Ed $(r=-0.42)$, suggesting that neutral or double-sided reflections, which combine negative and positive aspects of the target behavior, were related to reduced ongoing drug use at follow-up. In addition, REF was positively associated with DUDIT-Et $(r=0.40)$, DASEpos $(r=0.40)$, DASEneg $(r=$ $0.48)$, and DASEcrav $(r=0.40)$. This indicated that neutral or double-sided reflections$\mathrm{REF}$ - also were related to treatment interest, and high self-efficacy when in a positive mood, negative mood, and state of craving. Figure 1 shows an overview of connections with more than $10 \%$ common variance between counselor behaviors and client outcomes.

\section{Client In-session Language and Substance Use-Related Outcomes}

All associations between counselor in-session language and client substance use-related outcomes are shown in Table 1 . The quantity of CT was positively associated with DUDITEn $(r=0.44)$ and the motivational index, $(r=0.42)$, suggesting that utterances favoring change were related to perceptions of negative aspects of drug use and motivation to change at followup. Quantity of CT was also negatively associated with DASEphy $(r=-0.33)$ indicating that more change talk was related to lower self-efficacy regarding abstaining from drugs in

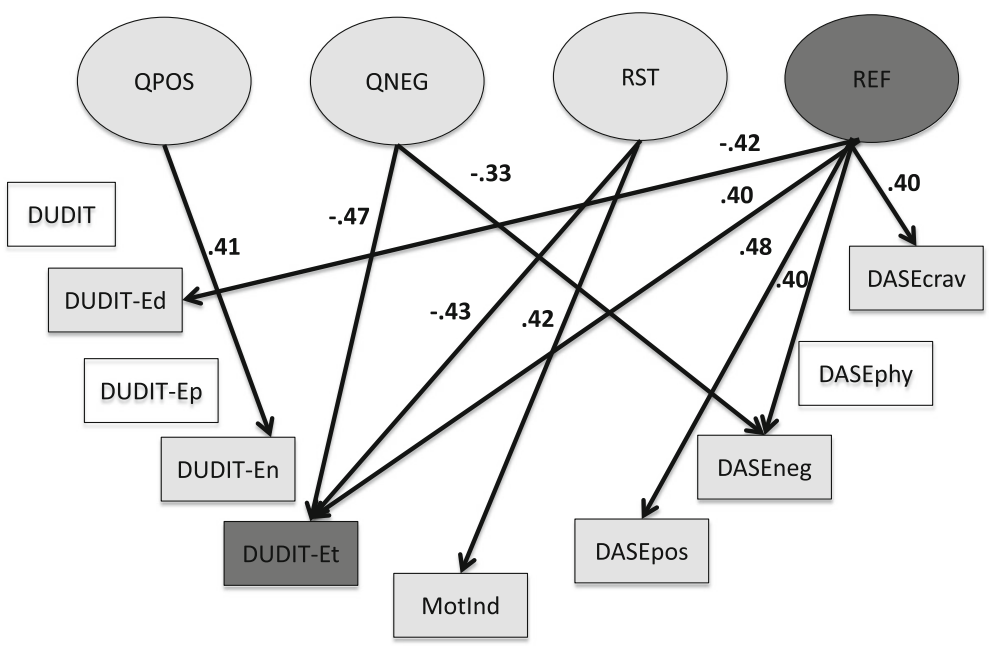

Fig. 1 Counselor verbal behaviors and client outcomes at 3-month follow-up, where more than $10 \%$ common variance occurred, based on squared Pearson correlations. Shading reflects number of connections with over $10 \%$ common variance, with light gray variables indicating 1-2 connections and dark gray variables indicating 3-5 connections. Non-shaded variables had no connections with any other variables. See Table 1 for abbreviation key 


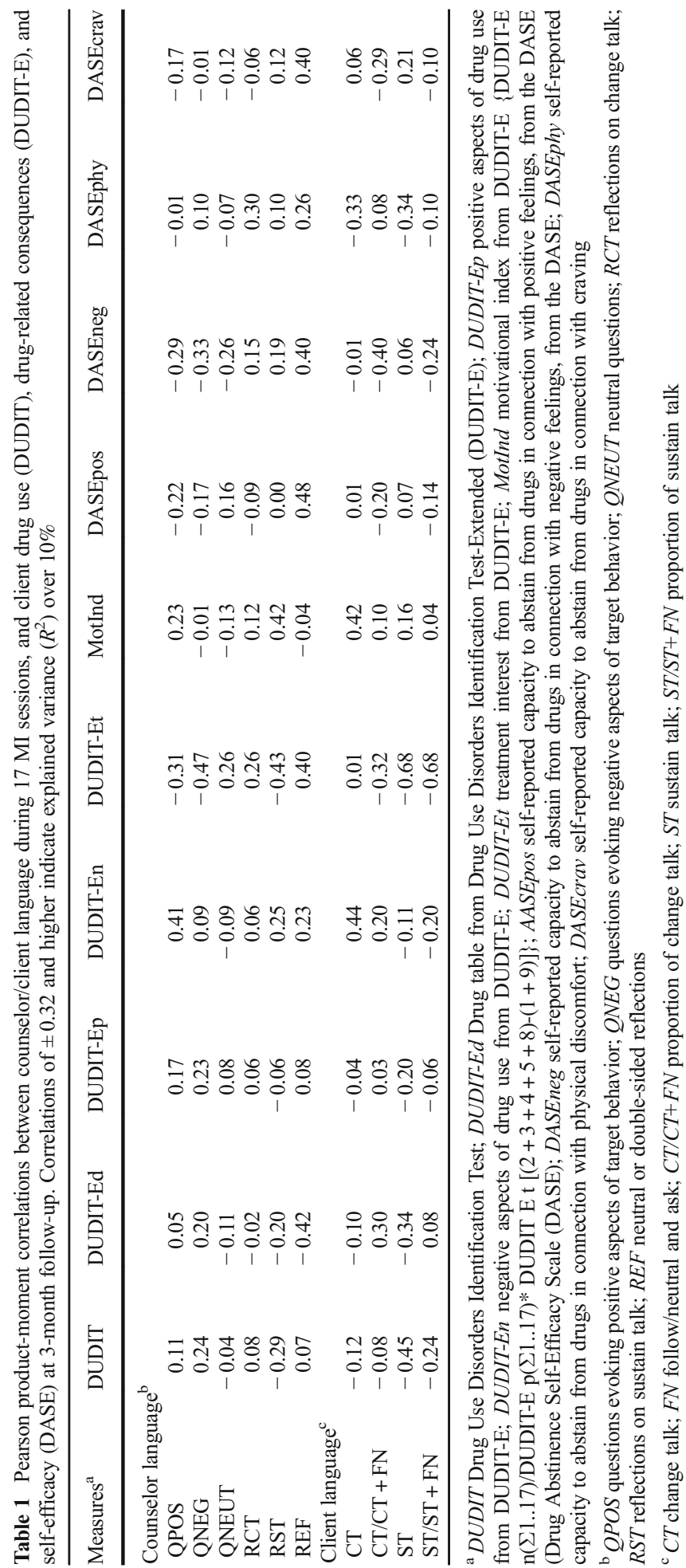


connection with physical discomfort. In addition, a negative correlation between proportion of CT and DASEneg $(r=-0.40)$ showed that participants who talked about changing their behavior also reported low self-efficacy with regard to abstaining from drugs in connection with negative feelings.

Furthermore, quantity of ST was negatively associated with DUDIT $(r=-0.45)$, and DUDITEd $(r=-0.34)$ scores, indicating that the more participants talked about sustaining their drug use, the lower their level of problematic drug use (DUDIT), and current drug consumption (DUDIT-Ed) at follow-up. Both quantity $(r=-0.68)$ and proportion $(r=-0.68)$ of ST showed negative correlations with DUDIT-Et suggesting that high sustain talk was associated with low interest in treatment. A negative correlation between quantity of ST and DASEphy $(r=-0.34)$ also showed that high sustain talk was related to lower capacity with regard to abstaining from drugs in connection with physical discomfort. Figure 2 shows an overview of connections with more than $10 \%$ common variance between client verbal behaviors and client outcomes.

\section{Counselor and Client In-session Language}

The transition analysis included 1907 counselor-to-client transitions from 24 sessions (see Table 2). We found that questions evoking positive aspects of drug use (QPOS) were about 13 times more likely than chance to be followed by ST. In contrast, questions evoking negative aspects of drug use (QNEG) were over six times more likely than chance to be followed by CT. Both QPOS and QNEG were almost six times less likely to be followed by FN. When a counselor reflected on change talk (RCT), clients were almost 14 times more likely than chance to respond with more CT, and clients were almost 56 times more likely than chance to respond to sustain talk reflections (RST) with ST. Transitions from counselors' neutral behaviors (QNEUT and REF) to clients' FN utterances were significantly more likely than chance, whereas transitions to CT or ST were less likely than chance.

In summary, CT was significantly more likely than chance following QNEG and RCT but less likely following RST, while ST was significantly more likely following QPOS and RST.

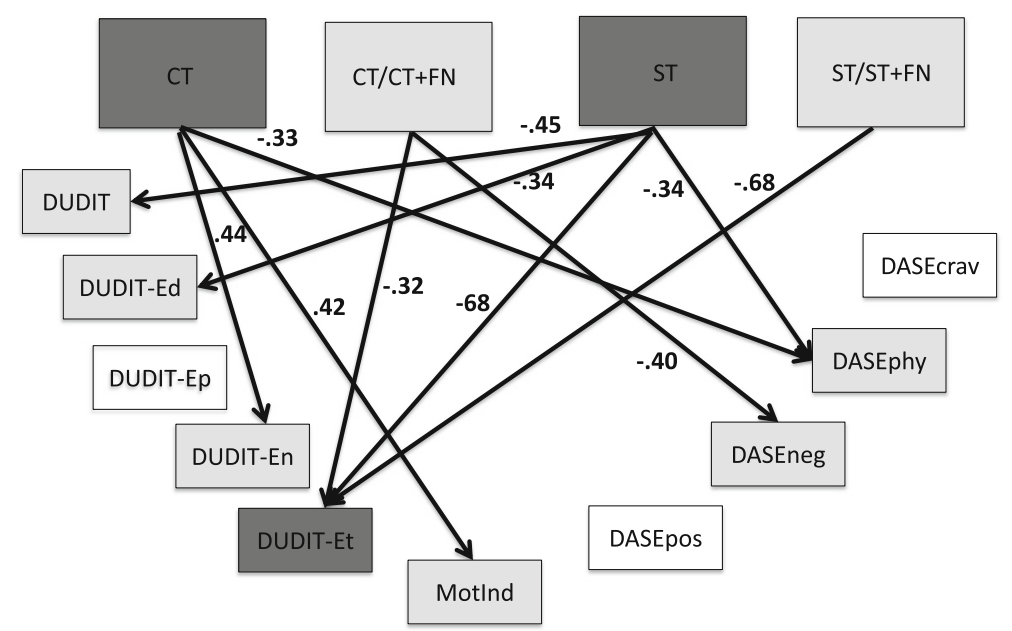

Fig. 2 Client verbal behaviors and client outcomes at 3-month follow-up, where more than $10 \%$ common variance occurred, based on squared Pearson correlations. See Fig. 1 for shading key. See Table 1 for abbreviation key 
Table 2 Counselor to client transition analysis

\begin{tabular}{lcccc}
\hline $\begin{array}{l}\text { Initial event } \rightarrow \text { subsequent } \\
\text { event }\end{array}$ & $\begin{array}{c}\text { Observed } \\
\text { frequency }\end{array}$ & $\begin{array}{c}\text { Expected } \\
\text { frequency }\end{array}$ & $\begin{array}{l}\text { Transition } \\
\text { probability }\end{array}$ & OR $(95 \%$ CI $)$ \\
\hline QPOS $\rightarrow$ FN & 21 & 44.40 & 0.34 & $0.18(0.11 ; 0.32)$ \\
QPOS $\rightarrow$ CT & 18 & 13.47 & 0.30 & $1.50(0.86 ; 2.63)$ \\
QPOS $\rightarrow$ ST & 22 & 3.14 & 0.36 & $13.14(7.42 ; 23.25)$ \\
QNEG $\rightarrow$ FN & 37 & 76.42 & 0.35 & $0.18(0.12 ; 0.27)$ \\
QNEG $\rightarrow$ CT & 64 & 23.18 & 0.61 & $6.32(4.20 ; 9.51)$ \\
QNEG $\rightarrow$ ST & 4 & 5.40 & 0.04 & $0.72(0.26 ; 2.00)$ \\
QNEUT $\rightarrow$ FN & 495 & 446.17 & 0.81 & $1.88(1.49 ; 2.38)$ \\
QNEUT $\rightarrow$ CT & 104 & 135.33 & 0.17 & $0.63(0.49 ; 0.81)$ \\
QNEUT $\rightarrow$ ST & 14 & 31.50 & 0.02 & $0.34(0.19 ; 0.60)$ \\
RCT $\rightarrow$ FN & 44 & 133.92 & 0.24 & $0.09(0.06 ; 0.13)$ \\
RCT $\rightarrow$ CT & 135 & 40.62 & 0.73 & $13.84(9.75 ; 19.66)$ \\
RCT $\rightarrow$ ST & 5 & 26.46 & 0.03 & $0.49(0.20 ; 1.22)$ \\
RST $\rightarrow$ FN & 9 & 7.95 & 0.25 & $0.12(0.06 ; 0.25)$ \\
RST $\rightarrow$ CT & 2 & $1.85^{\text {a }}$ & 0.06 & $0.20(0.05 ; 0.85)$ \\
RST $\rightarrow$ ST & 25 & 333.35 & 0.69 & $55.98(26.53 ; 118.12)$ \\
REF $\rightarrow$ FN & 418 & 101.11 & 0.91 & $5.16(3.66 ; 7.27)$ \\
REF $\rightarrow$ CT & 29 & 23.54 & 0.06 & $0.18(0.12 ; 0.27)$ \\
REF $\rightarrow$ ST & 11 & 327.53 & 0.02 & $0.39(0.20 ; 0.73)$ \\
Other $\rightarrow$ FN & 364 & 23.13 & 0.15 & $1.79(1.38 ; 2.32)$ \\
Other $\rightarrow$ CT & 69 & & 0.04 & $0.57(0.43 ; 0.75)$ \\
Other $\rightarrow$ ST & 17 & & & $0.67(0.39 ; 1.14)$ \\
\hline
\end{tabular}

OR odds ratio, CI confidence interval, QPOS positive questions, QNEG negative questions, QNEUT neutral questions, $R C T$ reflections on change talk, $R S T$ reflections on sustain talk, $R E F$ neutral or double-sided reflections, Other all other counselor utterances, $F N$ follow/neutral or "ask" statements by the client, $C T$ client change talk, ST client sustain talk

Both CT and ST were significantly less likely than chance following QNEUT and REF. Figure 3 shows an overview of the most significant associations between counselor and client in-session verbal utterances.

\section{Discussion}

This secondary analysis of the relationships between in-session counselor and client behaviors and self-reported client outcomes 3 months later shows some interesting significant
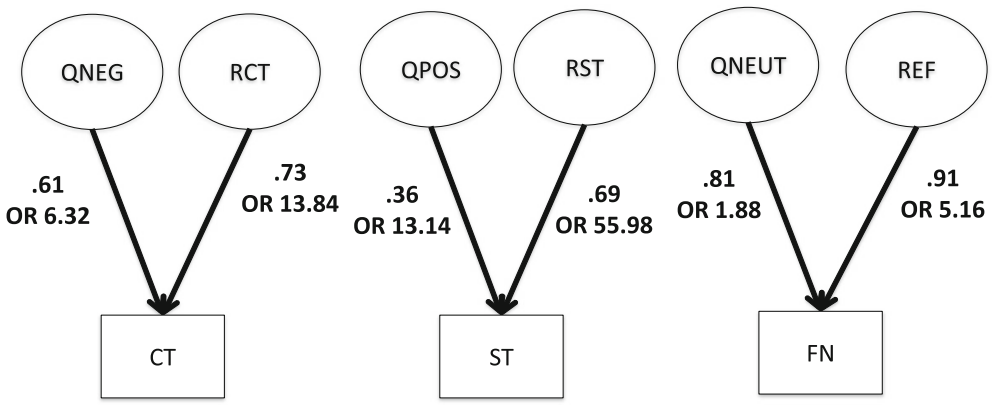

Fig. 3 Associations between counselor and client in-session language, expressed in transition probabilities and odds ratios. See Table 2 for abbreviation key 


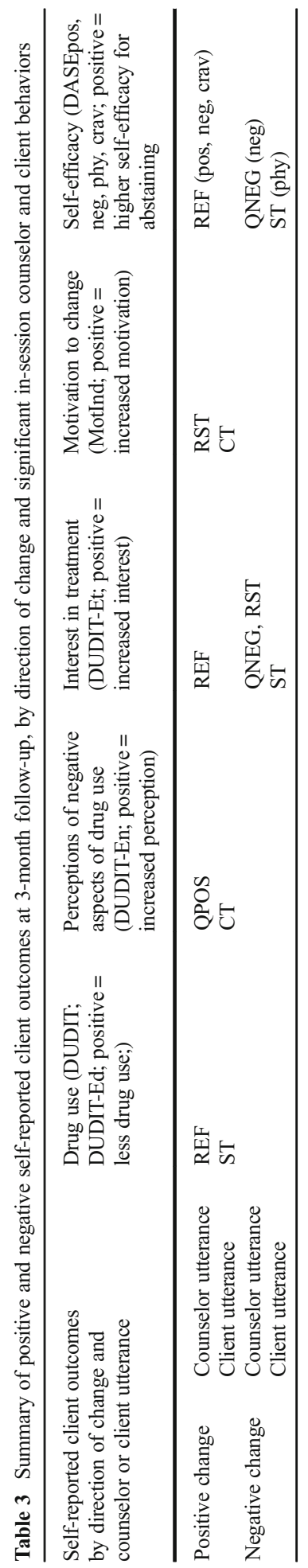


associations that serve to illustrate the complexity of the interactions between counselors, their clients, and clients' subsequent behavior. In order to facilitate interpretation of the results, the directions of client change at follow-up in relation to counselor and client utterances are summarized in Table 3.

Somewhat counter-intuitively, positive, health-oriented changes in client perceptions of the negative aspects of drug use were related to counselor questions about positive aspects of drug use as well as to client change talk, perhaps as a sort of client "righting reflex." Also, counterintuitively, health-oriented changes in drug use, interest in treatment, and self-efficacy were related to counselor reflections that were neutral or double-sided, as well as to client sustain talk (drug use). Motivation to change increased following counselor reflections of client sustain talk, as well as following client change talk. These relationships are partly understandable but partly counter to the existing research literature as well as being counter-intuitive. The picture becomes more complex when negative changes are examined: interest in treatment at follow-up was lower following counselor questions on negative aspects of drug use but, understandably, following reflections of sustain talk. Self-efficacy regarding abstaining from drugs in the presence of negative feelings decreased following counselor questions on negative aspects of drug use, possibly reflecting client concern over management of negative emotions without access to the self-medicating function of drugs. Self-efficacy in the presence of physical discomfort likewise decreased following client sustain talk, perhaps reflecting a similar mechanism of anxiety over the absence of an effective coping strategy that could replace drug use.

Two of our original hypotheses were nonetheless supported, namely that client change talk would be significantly associated with motivation to change, and that counselor behaviors focusing on client change talk would more frequently precede client change talk than counselor behaviors focusing on client sustain talk. The hypothesis that counselor focus on client change talk would be associated with client self-efficacy for abstaining from substance use was not supported. These findings raise questions regarding what might be happening for drug detox inpatients, who are experiencing vulnerable physical and emotional states as their neurological systems recuperate from drug intake and they find themselves in limbo, as hospital patients with a very brief respite before they are released back into their usual environment.

Detox inpatients may be significantly relieved at MI-counselors' non-confrontative approach, and particularly appreciate neutral reflections or double-sided ones juxtaposing positive and negative aspects of their ambivalence towards change. From the MI-perspective of four processes in counseling (Miller and Rollnick 2013), it may be helpful to recall that detox inpatients may need quite a lot of engagement efforts on the parts of counselors in order to establish a working alliance with patients, before it is possible to focus on a specific target behavior, much less evoke motivation for change and plan for specific changes. A study regarding the role of auricular acupuncture, a non-verbal treatment supporting nonconfrontative engagement (Berman and Lundberg 2002), in drug detox systems found that patients who had received acupuncture were $30 \%$ less likely to be re-admitted to detox in the first 6 months after treatment (Shwartz et al. 1999). Offering a single session of MI, while evidently producing some significant effects and associations, may be enough to raise patients' trust in the treatment system and hope for their own lives, but not quite enough to produce miracles - at least not during the relatively short follow-up period of 3 months. This proposition is supported by a randomized controlled trial evaluating two sessions of MI for psychiatric patients with substance use issues who were admitted to a psychiatric emergency unit, where the 1-year follow-up showed no differences between intervention and control groups, but the 
2-year follow-up showed significantly fewer substance use days for the intervention group but more substance use days for the control group, compared to the baseline level (Bagoien et al. 2013).

A brief review of prior research shows that, generally speaking, both in-session client and counselor verbal behavior contribute to behavioral outcome, where clients' change talk is the principal mediator of their change, and counselors' lower use of MI-inconsistent behaviors is associated with better client outcomes (Apodaca and Longabaugh 2009). Earlier studies found that frequencies of client language in favor of change or status quo were associated with days abstinent at follow-up (Moyers et al. 2007a), and that the average strength of patients' expressed ability to change predicted reductions in alcohol consumption, although counselor behaviors were not predictive of client outcome (Gaume et al. 2008a). A study among adolescents found that a high frequency of clients' in-session expressed reasons in favor of change predicted greater abstinence from alcohol or illicit substances, while expressed ability or desire in favor of status quo predicted less abstinence (Baer et al. 2008). More recently, a study among adolescents participating in a substance use prevention program found that the percentage of clients' change talk predicted reductions in marijuana use outcomes (Barnett et al. 2014a).

Counselor characteristics and behavior have shown variable associations with patient outcomes. A positive correlation has been identified between the way MI counselors are supported and the quality of their counseling, and change talk levels among heavy college student drinkers as well as their related drinking outcomes (Vader et al. 2010). A study focusing on college students who participated in mandated brief motivational interventions (BMIs) showed that therapists' MI-consistent behaviors were associated with both positive and negative change talk, possibly because therapists focused on exploring client ambivalence; however, therapist MI-consistent language did not show significant associations with client outcome, although therapist encouragement of client self-exploration was associated with a lower number of heavy drinking days (Apodaca et al. 2014). A study with non-treatmentseeking young men (military conscripts) who drank heavily and were randomized to BMI or a control group showed that outcomes were better when counselors were male, more experienced, had more positive attitudes and higher expectancies from (BMI) and better MI skills; however, fewer MI-consistent in-session behaviors were associated with better outcomes (Gaume et al. 2014). The percentage of counselor reflections on client change talk were also found to positively influence lower use of marijuana among clients (Barnett et al. 2014a, b).

Associations between therapists' MI skills and clients' in-session verbal behaviors have also been investigated. An initial landmark study found that therapist behavior that adhered to MI was more likely to be followed by client language that favored behavior change (Moyers and Martin 2006). Also, MI non-adherent behavior was more likely to be followed by client language that favored status quo. A sequential analysis of counselor and client behaviors showed that MI-consistent counselor behavior was more likely to be followed by client change talk, a finding that our results also clearly support; a conversational mutuality was also identified, where client exploration of change generated more MI-consistent counselor behavior (Gaume et al. 2008b).

A recent analysis of the role of reflections in catalyzing change talk indicated that reflections towards change (positive reflections) increased immediately following change talk by 11 times whereas reflections away from change (negative reflections) stimulated counter change talk by a factor of 19 . To be more effective, MI counselors need to enact their awareness of the valence of their reflections such that they reframe clients' counter change talk utterances into positive reflections; this would mean, for example, reflecting a client statement of "I'm never going to be able to change" positively as "You're really making an effort" rather than negatively as "Change is really tough for you" (Barnett et al. 2014b). This 
research was most recently analyzed in a meta-analysis of the key elements of causality in the MI conversational model, indicating that counselor MI-consistent behavior correlated significantly with client change talk $(r=0.26)$; however, change talk was not found to influence behavioral outcome at follow-ups at the same time that "sustain" talk, maintaining behavioral status quo, was related to more negative outcomes ( $r=-0.24)$ (Magill et al. 2014).

Our motivation for conducting the secondary analysis that this study presents was based on the above promising evidence that counselor language significantly affects client language as well as, possibly, behavioral outcomes. We did indeed find several significant relationships between counselor language and client language, as well as relationships between counselor and client language and behavioral outcomes. A major strength of this study is that we were able to identify associations of the kind earlier reported, following a single session of MI among patients in the detox setting, despite our very small sample. We find these circumstances encouraging in terms of the possibility of effectively helping detox patients orient themselves despite their vulnerable state, with a brief empathic intervention. Nonetheless, our study suffers from several limitations that would need to be addressed in future research in the detox setting. Firstly, our sample was small and technical issues reduced the sample size for complete data even more. The study was conducted in 2006-2008, when hospital staff members were regularly using Dictaphones with small tapes in order to record medical journal entries as well as to record the MI sessions in the study. All Dictaphone recordings were converted into digital recordings, but some were of a quality too low to be transcribed. These obstacles may be a thing of the past given the advent of smartphones offering reliable and secure methods of recording. A second limitation concerned the SCOPE coding, which was a newly introduced method at our MI coding lab. SCOPE coders conducted a series of test sessions until they achieved adequate inter-rater reliability, but this procedure was not formally documented and may have resulted in lower intra-class correlation coefficients than the usual standard; however, the coders did test inter-rater reliability in relation to smoking cessation transcripts and these were satisfactory.

In conclusion, this study indicates some additional value for single-session MI in the drug detox setting, beyond the controlled outcomes reported earlier. Client change talk was significantly associated with motivation to change drug use behavior and counselor behaviors focusing on client change talk did more frequently precede client change talk. The processes ongoing in the counselor-client interaction in the detox setting, where the patient is subject to multiple physical and emotional stressors and increased vulnerability, are most likely quite complex. The single MI session may be more heavily focused on engagement processes, where counselor reflections that are neutral or double-sided may serve to enhance the patient experience of empathy and engagement, but later-after release from the detox unit-evoke client motivation to reduce drug use, engage in treatment, and increase self-efficacy. The processes initiated in the single MI session may indeed reverberate long after the detox stay. Future research should investigate the possible additional effects of two MI sessions, increase sample size, and extend follow-up to at least 2 years after release.

Acknowledgements We acknowledge the participation of the patients in the study, the staff MI-counselors, and Beatrice Biguet for transcription of the counseling sessions.

Funding sources This work was supported by Swedish Society of Medicine Funds (grant number SLS252831), the Swedish Drug Policy Coordinator (grant number MOB 238-2006-14), and the Swedish Research Council (grant number K2012-61P-22, 132-01-6). The funding organizations had no role in the conception of the study, in the analyses of the data, in the writing of the manuscript, nor in the decision to submit it for publication. 


\section{Compliance with Ethical Standards}

Conflict of Interest The authors AHB, ND, HK, and UH declare that they have no conflict of interest. Author LF declares that he owns a company providing MI coding services; however, this was not the company that provided coding services for the work reported in this study.

Open Access This article is distributed under the terms of the Creative Commons Attribution 4.0 International License (http://creativecommons.org/licenses/by/4.0/), which permits unrestricted use, distribution, and reproduction in any medium, provided you give appropriate credit to the original author(s) and the source, provide a link to the Creative Commons license, and indicate if changes were made.

\section{References}

Apodaca, T. R., \& Longabaugh, R. (2009). Mechanisms of change in motivational interviewing: a review and preliminary evaluation of the evidence. Addiction, 104, 705-715.

Apodaca, T. R., Borsari, B., Jackson, K. M., Magill, M., Longabaugh, R., Mastroleo, N. R., \& Barnett, N. P. (2014). Sustain talk predicts poorer outcomes among mandated college student drinkers receiving a brief motivational intervention. Psychology of Addictive Behaviors, 28(3), 631.

Baer, J. S., Beadnell, B., Garrett, S. B., Hartzler, B., Wells, E. A., \& Peterson, P. L. (2008). Adolescent change language within a brief motivational intervention and substance use outcomes. Psychology of Addictive Behaviors, 22, 570 575.

Bagoien, G., Bjørngaard, J. H., Østensen, C., Reitan, S. K., Romundstad, P., \& Morken, G. (2013). The effects of motivational interviewing on patients with comorbid substance use admitted to a psychiatric emergency unita randomised controlled trial with two year follow-up. BMC Psychiatry, 13(1), 1.

Bakeman, R., \& Quera, V. (2011). Sequential analysis and observational methods for the behavioral sciences. New York: Cambridge University Press.

Bakeman, R., \& Quera, V. (2015). GSEQ 5.1. Software for the analysis of interaction sequences. Retrieved May 15, 2015 from http://www2.gsu.edu/ psyrab/gseq/Download.html.

Bakeman, R., Quera, V., Gnisci, A. (2009). Observer agreement for timed-event sequential data: a comparison of time-based and event-based algorithms. Behavior Research Methods, 41(1), 137-147.

Barnett, E., Moyers, T. B., Sussman, S., Smith, C., Rohrbach, L. A., Sun, P., \& Spruijt-Metz, D. (2014a). From counselor skill to decreased marijuana use: does change talk matter? Journal of Substance Abuse Treatment, 46(4), 498-505.

Barnett, E., Spruijt-Metz, D., Moyers, T. B., Smith, C., Rohrbach, L. A., Sun, P., \& Sussman, S. (2014b). Bidirectional relationships between client and counselor speech: the importance of reframing. Psychology of Addictive Behaviors, 28(4), 1212.

Berman, A. H., \& Lundberg, U. (2002). Auricular acupuncture in prison psychiatric units: a pilot study. Acta Psychiatrica Scandinavica, 106(Suppl. 412), 152-157.

Berman, A. H., Bergman, H., Palmstierna, T., \& Schlyter, F. (2005). Evaluation of the Drug Use Disorders Identification Test (DUDIT) in criminal justice and detoxification settings and in a Swedish population sample. European Addiction Research, 11, 22-31.

Berman, A. H., Palmstierna, T., Källmén, H., \& Bergman, H. (2007). The self-report Drug Use Disorders Identification Test-Extended (DUDIT-E): reliability, validity, and motivational index. Journal of Substance Abuse Treatment, 32, 357-369.

Berman, A. H., Forsberg, L., Durbeej, N., Källmén, H., \& Hermansson, U. (2010). Single-session motivational interviewing for drug detoxification inpatients: effects on self-efficacy, stages of change and substance use. Substance Use \& Misuse, 45, 384-402.

Cicchetti, V. (1994). Guidelines, criteria and rules of thumb for evaluating normed and standardized assessment instruments in psychology. Psychological Assessment, 6, 284-290.

Cohen, J. (1988). Statistical power analysis for the behavioral sciences (2nd ed.). Hillsdale, NJ: Erlbaum.

DiClemente, C., Carbonari, J. P., Montgomery, R. P. G., \& Hughes, S. O. (1994). The Alcohol Abstinence SelfEfficacy Scale. Journal of Studies on Alcohol, 55, 141-148.

Dobber, J., van Meijel, B., Barkhof, E., Scholte op Reimer, W., Latour, C., Peters, R., \& Linszen, D. (2015). Selecting an optimal instrument to identify active ingredients of the motivational interviewing-process. Journal of Psychosomatic Research, 78(3), 268-276. https://doi.org/10.1016/j.jpsychores.2014.10.010.

Forsberg, L., Lindqvist, H., Lindroos, N., Hellberg, C., \& Forsberg, L. (2014). Kodningsmanual for sekventiell kodning av motiverande samtal för att observera processuella utbyten av yttranden. [Swedish translation and processing of Martin T, Moyers TB, Houck J, Christopher P, Miller W, (2005) Motivational Interviewing Sequential Code for Observing Process Exchanges (MI-SCOPE) Coder's Manual. Albuquerque, NM: 
University of New Mexico, Center on Alcoholism, Substance Abuse, and Addictions (CASAA).] Karolinska Institutet: Stockholm. (In review)

Gaume, J., Gmel, G., \& Daeppen, J. B. (2008a). Brief alcohol interventions: do counsellors' and patients' communication characteristics predict change?. Alcohol and Alcoholism, 43, 62-69.

Gaume, J., Gmel, G., Faouzi, M., \& Daeppen, J. B. (2008b). Counsellor behaviours and patient language during brief motivational interventions: a sequential analysis of speech. Addiction, 103(11), 1793-1800.

Gaume, J., Magill, M., Longabaugh, R., Bertholet, N., Gmel, G., \& Daeppen, J.-B. (2014). Influence of counselor characteristics and behaviors on the efficacy of a brief motivational intervention for heavy drinking in young men - a randomized controlled trial. Alcoholism: Clinical and Experimental Research, 38(7), 2138-2147. https://doi.org/10.1111/acer.12469.

Glynn, L. H., Hallgren, K. A., Houck, J. M., \& Moyers, T. B. (2012). CACTI: free, open-source software for the sequential coding of behavioral interactions. PLoS One, 7(7), e39740. https://doi.org/10.1371/journal. pone.0039740.

Hildebrand, M. (2015). The psychometric properties of the Drug Use Disorders Identification Test (DUDIT): a review of recent research. Journal of Substance Abuse Treatment, 53, 52-59.https://doi.org/10.1016/j. jsat.2015.01.008.

Magill, M., Gaume, J., Apodaca, T. R., Walthers, J., Mastroleo, N. R., Borsari, B., \& Longabaugh, R. (2014). The technical hypothesis of motivational interviewing: a meta-analysis of MI's key causal model. Journal of Consulting and Clinical Psychology, 82(6), 973.

Martin, T., Moyers, T. B., Houck, J. M., Christopher, P. J., \& Miller, W. R. (2005). Motivational interviewing sequential code for observing process exchanges (MI-SCOPE) coder's manual. Albuquerque, NM: Unpublished manuscript, Center on Alcoholism, Substance Abuse, and Addictions, University of New Mexico.

McKiernan, P., Cloud, R., Patterson, D. A., Golder, S., \& Besel, K. (2011). Development of a brief abstinence self-efficacy measure. Journal of Social Work Practice in the Addictions, 11(3), 245-253.

Miller, W. R., \& Moyers, T. B. (2015). The forest and the trees: relational and specific factors in addiction treatment. Addiction, $110(3), 401-413$.

Miller, W. R., \& Rollnick, S. (2013). Motivational interviewing: helping people change (3rd ed.). New York: Guilford Press.

Miller, W. R., \& Rose, G. S. (2009). Toward a theory of motivational interviewing. American Psychologist, 64(6), 527.

Moyers, T. B., \& Martin, T. (2006). Therapist influence on client language during motivational interviewing sessions. Journal of Substance Abuse Treatment, 30, 245-251.

Moyers, T. B., Martin, T., Catley, D., Harris, K. J., \& Ahluwalia, J. S. (2003). Assessing the integrity of motivational interviewing interventions: reliability of the motivational interviewing skills code. Behavioural and Cognitive Psychotherapy, 31(02), 177-184.

Moyers, T. B., Martin, T., Christopher, P. J., Houck, J. M., Tonigan, J. S., \& Amrhein, P. (2007a). Client language as a mediator of motivational interviewing efficacy: where is the evidence? Alcoholism: Clinical and Experimental Research, 31(s3), 40s-47s.

Moyers, T. B., Martin, T., Manual, J. K., Miller, W. R., \& Ernst, D. (2007b). Revised Global Scales: Motivational Interviewing Treatment Integrity 3.0 (MITI 3.0). Substance Abuse and Addictions (CASAA). University of New Mexico, Center on Alcoholism. Retrieved on January 7, 2018 at https://casaa.unm.edu/download/miti3.pdf.

Moyers, T. B., Martin, T., Houck, J. M., Christopher, P. J., \& Tonigan, J. S. (2009). From in-session behaviors to drinking outcomes: a causal chain for motivational interviewing. Journal of Consulting and Clinical Psychology, 77(6), 1113.

Pace, B. T., Dembe, A., Soma, C. S., Baldwin, S. A., Atkins, D. C., \& Imel, Z. E. (2017). A multivariate metaanalysis of motivational interviewing process and outcome. Psychology of Addictive Behaviors, 31 (5), 524 533. https://doi.org/10.1037/adb0000280.

Quera, V., Bakeman, R., Gnisci, A. (2007). Observer agreement for event sequences: methods and software for sequence alignment and reliability estimates. Behavior Research Methods, 39(1), 39-49.

Schilling, R. F., El-Bassel, N., Finch, J. B., Roman, R. J., \& Hanson, M. (2002). Motivational interviewing to encourage self-help participation following alcohol detoxification. Research on Social Work Practice, 12(6), 711-730. https://doi.org/10.1177/104973102237469.

Shwartz, M., Saitz, R., Mulvey, K., \& Brannigan, P. (1999). The value of acupuncture detoxification programs in a substance abuse treatment system. Journal of Substance Abuse Treatment, 17(4), 305-312.

Vader, A. M., Walters, S. T., Prabhu, G. C., Houck, J. M., \& Field, C. A. (2010). The language of motivational interviewing and feedback: counselor language, client language, and client drinking outcomes. Psychology of Addictive Behaviors, 24(2), 190-197. 